DELAYED PROTONS FOLLOWING THE DEGAY OF ARGON-33

J. C. Hardy and R. I. Verrall 
DELAYED PROTONS FOLLOWING THE DEGAY OF ARGON-33

J. C. HARdy and R. I. Verrall 


\title{
DELAYED PROTONS FOLLOWING THE DECAY OF ARGON-33
}

\author{
J. C. Hardy and R. I. Verrall \\ Foster Radiation Laboratory, McGill University, Montreal, P.Q. \\ Received November 27, 1964
}

ABSTRACT

The delayed-proton precursor ${ }^{33} \mathrm{Ar}$ has been produced by proton bombardment of a lithium chloride target in the internal beam of the McGill synchrocyclotron. The half-life of ${ }^{33} \mathrm{Ar}$ was measured to be $(178 \pm 10)$ msec. To explain the delayed proton spectrum, two new levels, at 5.55 and $7.55 \mathrm{MeV}$, in ${ }^{33} \mathrm{Cl}$ are proposed. It is also proposed that the former is the expected first $T=3 / 2$ state in that nucleus.

Delayed protons have been observed following the decay of ${ }^{33} \mathrm{Ar}$. The only previous reports of this nuclide are brief mentions by Reeder et al. (1964) and by us (Hardy and Verrall 1964a) in simultaneous publications that were devoted mainly to another delayed proton precursor, ${ }^{37} \mathrm{Ca}$. We now report our results in detail; they differ somewhat from those of Reeder et al.

The measurements were carried out as in previous experiments in the present series (e.g. McPherson et al. 1964), using thin targets bombarded in the circulating proton beam of the McGill synchrocyclotron and observed by surface-barrier silicon detectors. Figure 1 shows the delayed proton spectrum from a chlorine target $\left(2.3 \mathrm{mg} / \mathrm{cm}^{2} \mathrm{LiCl}\right.$ vacuum evaporated on a $200 \mu \mathrm{g} / \mathrm{cm}^{2}$ gold backing). Shown as an inset in Fig. 1 is the spectrum of delayed protons obtained from a sulphur target, which is taken from the work of Hardy and Verrall $(1964 b)$. We now argue that in the main spectrum only the two peaks located at channels 47 and 98 can be said definitely to follow the decay of ${ }^{33} \mathrm{Ar}$. Two other peaks, at channels 67 and 114, are too small to be identified positively, while all other peaks are attributed to ${ }^{29} \mathrm{~S}$ and ${ }^{25} \mathrm{Si}$.

The peak in channel 105 of the main spectrum was shown to follow the decay of ${ }^{29} \mathrm{~S}$ by means of the following arguments. Its production threshold was found to be $(50 \pm 5) \mathrm{MeV}$, which agrees with the calculated threshold for the reaction ${ }^{35} \mathrm{Cl}(\mathrm{p}, \alpha 3 \mathrm{n}){ }^{29} \mathrm{~S}$. Also, the measured energy of this peak is the same as that previously found (cf. inset) for the principal proton group following the decay of ${ }^{29} \mathrm{~S}$. By comparison with the inserted spectrum, several other peaks in the main spectrum can be identified, and these are seen to appear with the relative intensities expected.

It can be seen that the predominant peak in channel 47 was not observed following proton bombardment of sulphur, and consequently the nuclide responsible for the activity must be an isotope of argon. A measurement of the threshold for its production was difficult to obtain because of the relatively high $\beta$ background; however, an approximate value of $(39 \pm 5) \mathrm{MeV}$ was determined. The argon isotope must then be ${ }^{33} \mathrm{Ar}$ since the observed threshold is consistent only with the reaction ${ }^{35} \mathrm{Cl}(\mathrm{p}, 3 \mathrm{n}){ }^{33} \mathrm{Ar}$, whose calculated threshold is $37.8 \mathrm{MeV}$. A decay curve of the net area under this peak is shown in Fig. 2, and gives the half-life as $(178 \pm 10) \mathrm{msec}$. 


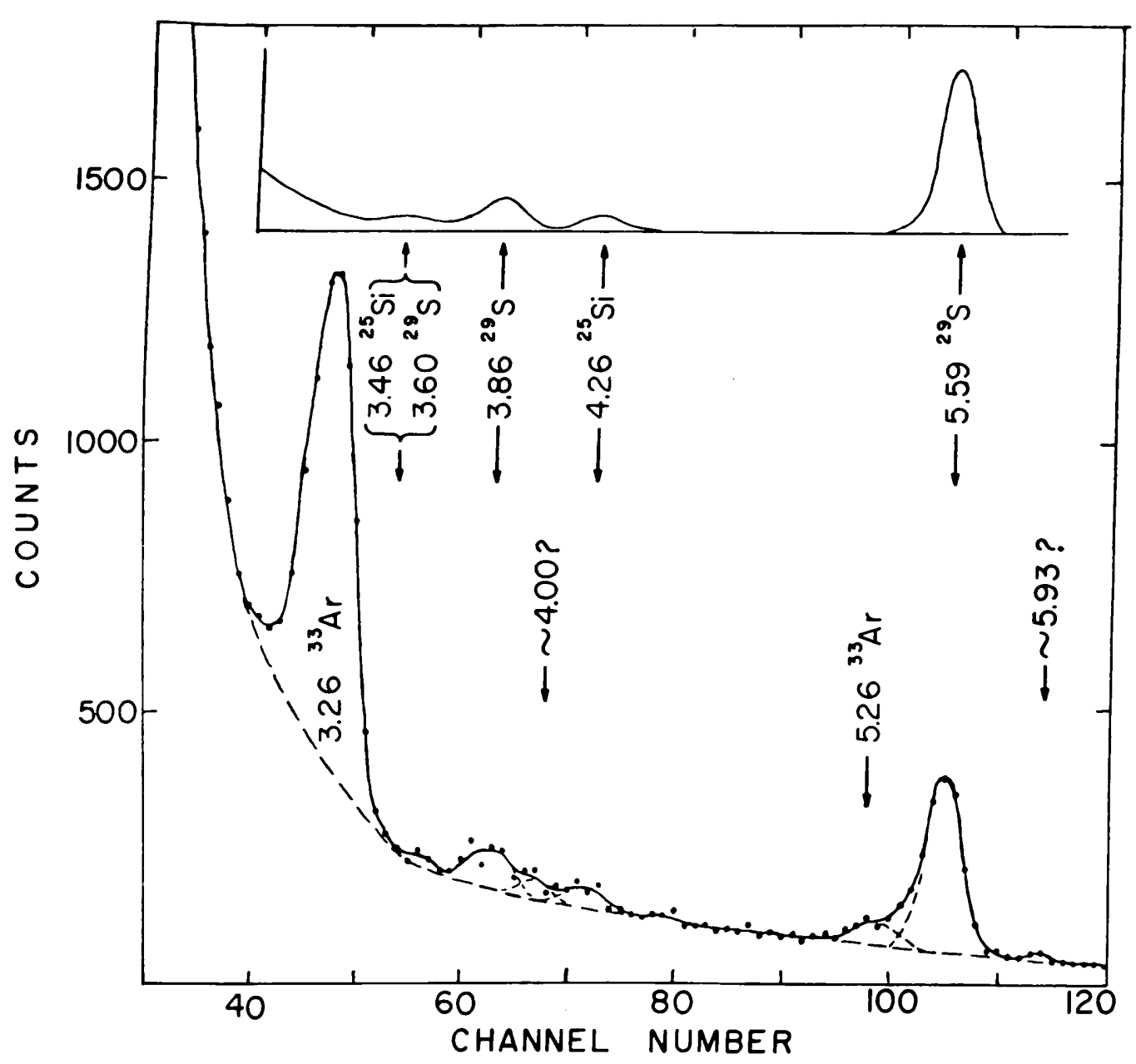

Fici. 1. Spectrum of delayed protons from a chlorine target. The energy of each proton peak is shown in $\mathrm{Mel}$, corrected to center of mass. The energies of the two peaks of uncertain origin have been calculated for mass 33 . The inset shows the spectrum from a sulphur target and has been normalized so that the peak height at $5.59 \mathrm{MeV}$ is the same in both spectra.

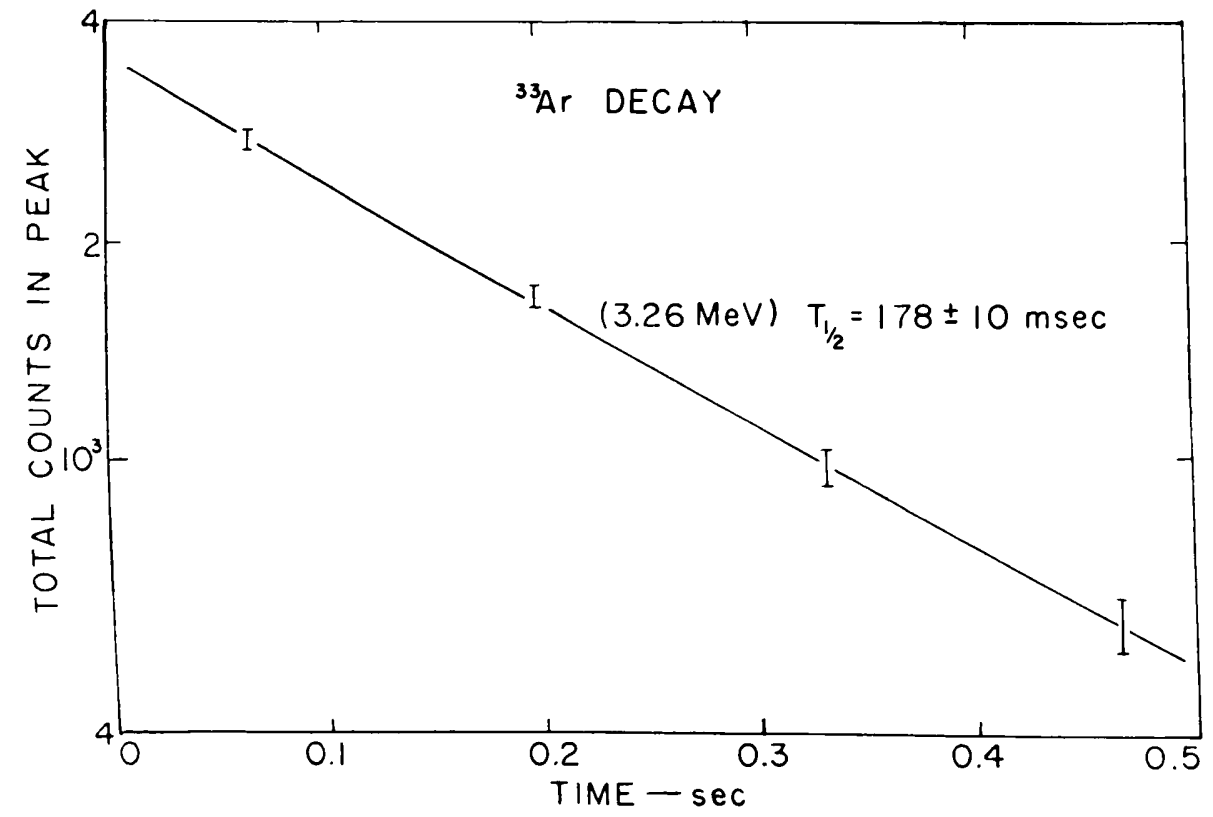

Fir. 2. Time decay of the net area under the main peak of Fig. 1, showing that the half-life of ${ }^{33} . \mathrm{Ar}$ is $(17 \mathrm{~s} \pm 10) \mathrm{msec}$. 
From lifetime and threshold measurements, the peak in channel 98 (Fig. 1) appears also to be associated with ${ }^{33} \mathrm{Ar}$; its intensity is about $4 \%$ of that of the main peak $(3.26 \mathrm{MeV})$ of the ${ }^{33} \mathrm{Ar}$ spectrum.

The energies, corrected to center of mass, of the two peaks assigned to ${ }^{33} \mathrm{Ar}$ are $(5.26 \pm 0.1)$ and $(3.26 \pm 0.05) \mathrm{MeV}$, as is indicated in Fig. 1. Figure 3 shows the proposed decay scheme of ${ }^{33} \mathrm{Ar}$ and includes two new levels at $(7.55 \pm$ $0.1)$ and $(5.55 \pm 0.05) \mathrm{MeV}$ which are introduced to explain the two observed proton peaks. The first level is in an energy region not previously explored, and the second has apparently not been observed in proton-scattering experiments.

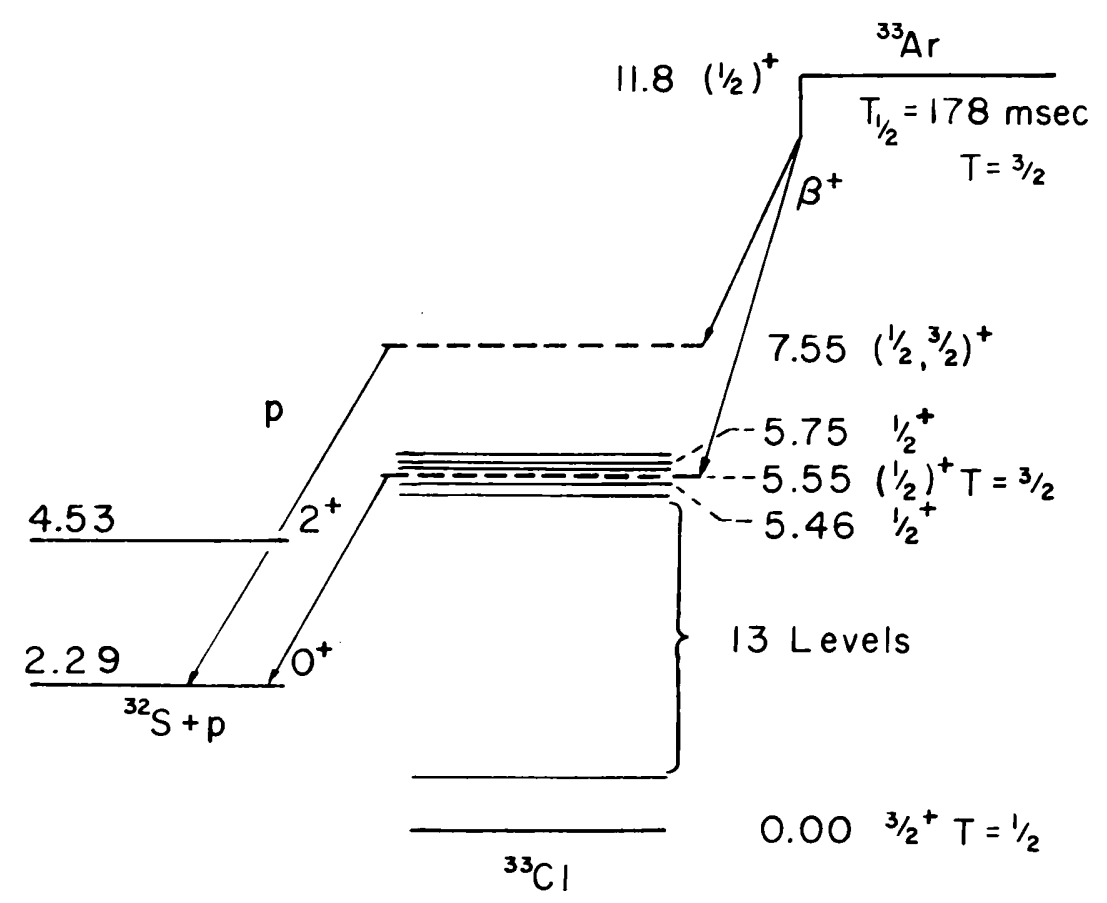

FIG. 3. Proposed decay scheme of ${ }^{33} \mathrm{Ar}$. Previously known levels, taken from the compilation of Endt and van der Leun (1962), are shown as solid lines.

As well as observing the peak at $3.26 \mathrm{MeV}$, Reeder et al. observed a peak at $(3.90 \pm 0.1) \mathrm{MeV}$ which they attributed to ${ }^{33} \mathrm{Ar}$. This could be the unidentified peak which we observed at about $4.0 \mathrm{MeV}$. Their measured spectrum does not extend to sufficiently high energies to include the ${ }^{33} \mathrm{Ar}$ peak at $5.26 \mathrm{MeV}$.

All known nuclei with 15 odd nucleons have an assigned spin parity of $\frac{1}{2}^{+}$, and for this reason, we have assigned $\frac{1}{2}+$ to ${ }^{33} \mathrm{Ar}$. On the basis of this assignment there are two known levels in ${ }^{33} \mathrm{Cl}$ which should be fed by allowed $\beta$ transitions and which should decay by emitting protons within the energy range of observation. Such proton groups (expected at 3.17 and $3.46 \mathrm{MeV}$ ) are certainly: not present with an intensity comparable to that of the observed $3.26-\mathrm{MeV}$ peak. This suggests that the level at $5.55 \mathrm{MeV}$ is fed by a superallowed $\beta^{+}$transition from the $T=3 / 2$ ground state of ${ }^{33} \mathrm{Ar}$, and is therefore the expected first $T=3 / 2$ state in ${ }^{33} \mathrm{Cl}$. This isotopic spin assignment would also account for the level's nonappearance in resonance data for proton scattering from ${ }^{32} \mathrm{~S}$. Using the semiempirical formula of Jaenecke (1960) for Coulomb energy differences, and assuming isobaric invariance, the expected energy of the first $T=3 / 2$ level was calculated to be $5.54 \mathrm{MeV}$, which is in good agreement with our experimental value. 